\title{
ggplot2 Compatible Quantile-Quantile Plots in $\mathbf{R}$
}

\author{
by Alexandre Almeida, Adam Loy, Heike Hofmann
}

\begin{abstract}
Q-Q plots allow us to assess univariate distributional assumptions by comparing a set of quantiles from the empirical and the theoretical distributions in the form of a scatterplot. To aid in the interpretation of Q-Q plots, reference lines and confidence bands are often added. We can also detrend the Q-Q plot so the vertical comparisons of interest come into focus. Various implementations of Q-Q plots exist in $\mathrm{R}$, but none implements all of these features. qqplotr extends ggplot2 to provide a complete implementation of $\mathrm{Q}-\mathrm{Q}$ plots. This paper introduces the plotting framework provided by qqplotr and provides multiple examples of how it can be used.
\end{abstract}

\section{Background}

Univariate distributional assessment is a common thread throughout statistical analyses during both the exploratory and confirmatory stages. When we begin exploring a new data set we often consider the distribution of individual variables before moving on to explore multivariate relationships. After a model has been fit to a data set, we must assess whether the distributional assumptions made are reasonable, and if they are not, then we must understand the impact this has on the conclusions of the model. Graphics provide arguably the most common way to carry out these univariate assessments. While there are many plots that can be used for distributional exploration and assessment, a quantilequantile (Q-Q) plot (Wilk and Gnanadesikan, 1968) is one of the most common plots used.

Q-Q plots compare two distributions by matching a common set of quantiles. To compare a sample, $y_{1}, y_{2}, \ldots, y_{n}$, to a theoretical distribution, a Q-Q plot is simply a scatterplot of the sample quantiles, $y_{(i)}$, against the corresponding quantiles from the theoretical distribution, $F^{-1}\left(F_{n}\left(y_{(i)}\right)\right)$. If the empirical distribution is consistent with the theoretical distribution, then the points will fall on a line. For example, Figure 1 shows two Q-Q plots: the left plot compares a sample drawn from a lognormal distribution to a lognormal distribution, while the right plot compares a sample drawn from a lognormal distribution to a normal distribution. As expected, the lognormal Q-Q plot is approximately linear, as the data and model are in agreement, while the normal Q-Q plot is curved, indicating disagreement between the data and the model.

Additional graphical elements are often added to Q-Q plots in order to aid in distributional assessment. A reference line is often added to a $\mathrm{Q}-\mathrm{Q}$ plot to help detection of departures from the proposed model. This line is often drawn either by tracing the identity line or by connecting two pairs of quantiles, such as the first and third quartiles, which is known as the $Q-Q$ line. Pointwise or simultaneous confidence bands can be built around the reference line to display the expected degree of sampling error for the proposed model. Such bands help gauge how troubling a departure from the proposed model may be. Figure 2 adds Q-Q lines and 95\% pointwise confidence bands to the $\mathrm{Q}-\mathrm{Q}$ plots in Figure 1. While confidence bands help analysts interpret Q-Q plots, this practice is less
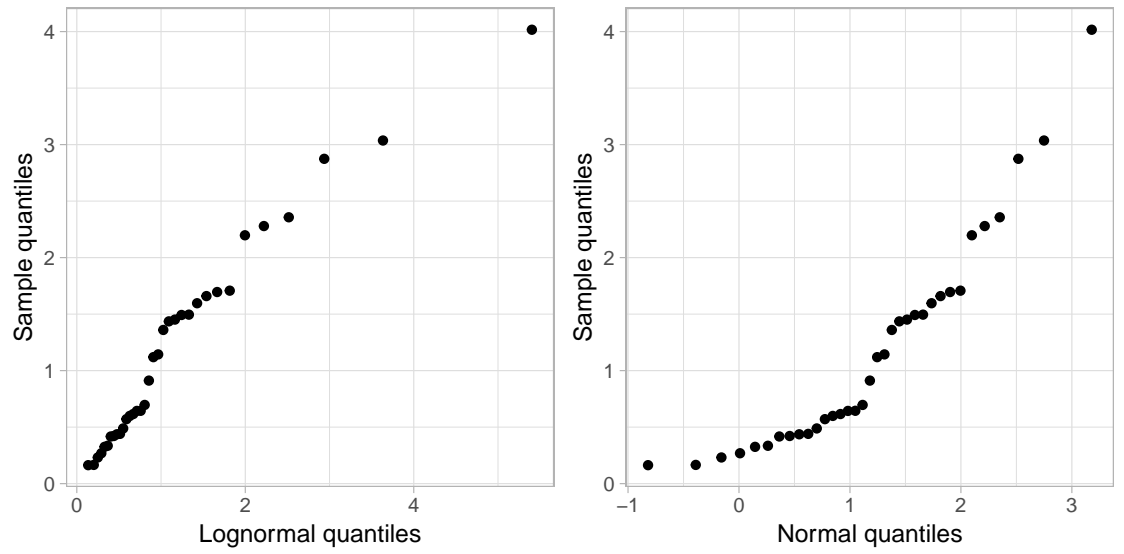

Figure 1: The left plot compares a sample of size $n=35$ drawn from a lognormal distribution to a lognormal distribution, while the right plot compares this sample to a normal distribution. The curvature in the normal Q-Q plot highlights the disagreement between the data and the model. 

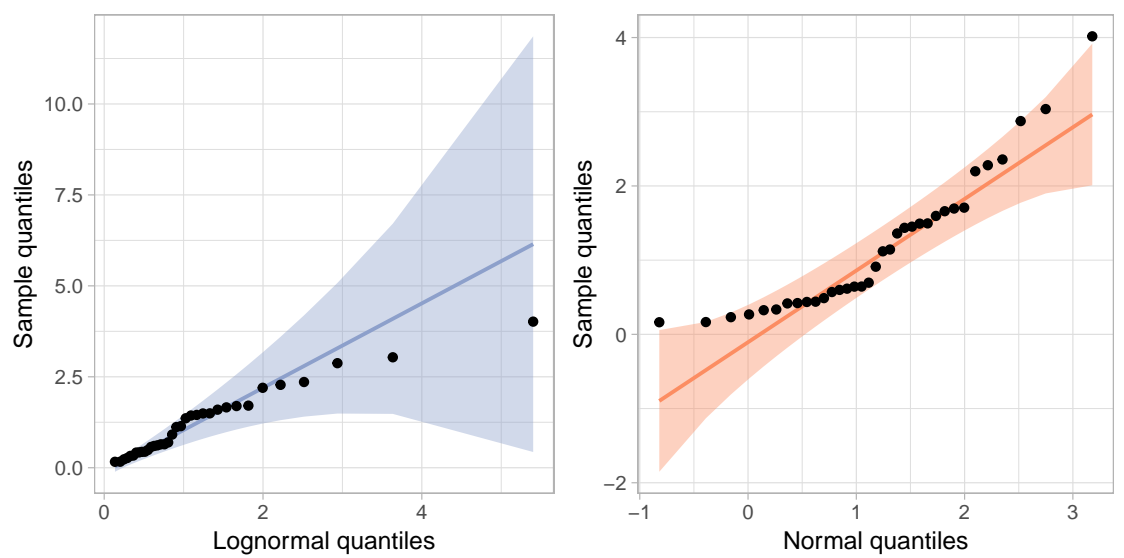

Figure 2: Adding reference lines and 95\% pointwise confidence bands to the Q-Q plots in Figure 1.
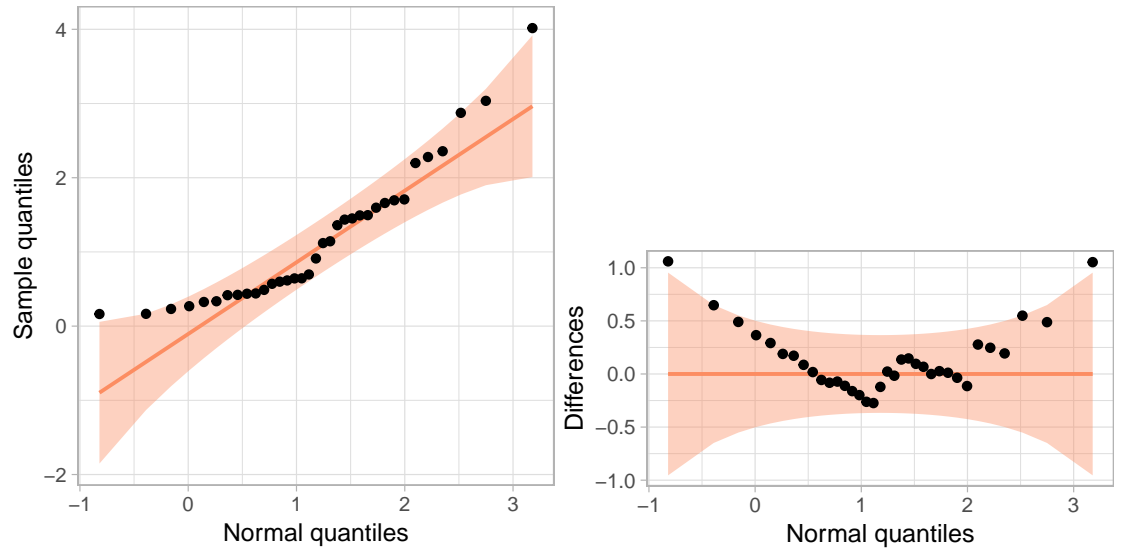

Figure 3: The left plot displays a traditional normal Q-Q plot for data simulated from a lognormal distribution. The right plot displays an adjusted detrended Q-Q plot of the same data, created by plotting the differences between the sample quantiles and the proposed model on the $y$-axis.

commonplace than it ought to be. One possible cause is that confidence bands are not implemented in all statistical software packages. Further, manual implementation can be tedious for the analyst, breaking the data-analytic flow-for example, a common simultaneous confidence band relies on an inversion of the Kolmogorov-Smirnov test.

Different orientations of Q-Q plots have also been proposed, most notably the detrended Q-Q plot. To detrend a Q-Q plot, the $y$-axis is changed to show the difference between the observed quantile and the reference line. Consequently, the $y$-axis represents agreement with the theoretical distribution. This makes the de-trended version of a Q-Q plot easier to process: cognitive research (Vander Plas and Hofmann, 2015; Robbins, 2005; Cleveland and McGill, 1984) suggests that onlookers have a tendency to intuitively assess the distance between points and lines based on the shortest distance (i.e., the orthogonal distance) rather than the vertical distance appropriate for the situation. In the de-trended Q-Q plot, the line to compare to points is rotated parallel to the $x$-axis, which makes assessing the vertical distance equal to assessing orthogonal distance. This is further investigated in Loy et al. (2016), who find that detrended Q-Q plots are more powerful than other designs as long as the $x$-and $y$-axes are adjusted to ensure that distances in the $x$ - and $y$-directions are on the same scale. This Q-Q plot design is called an adjusted detrended Q-Q plot. Without this adjustment to the range of the axes, ordinary detrended $Q-Q$ plots are produced, which were found to have lower power than the standard Q-Q plot in some situations (Loy et al., 2016), while the adjusted detrended Q-Q plots were found to be consistently more powerful. Figure 3 displays the normal Q-Q plot from Figure 2 along with its adjusted detrended version.

Various implementations of Q-Q plots exist in R. Normal Q-Q plots, where a sample is compared to the Standard Normal Distribution, are implemented using qqnorm and qqline in base graphics. qqplot provides a more general approach in base $\mathrm{R}$ that allows a specification of a second vector of quantiles, enabling comparisons to distributions other than a Normal. Similarly, the lattice package provides a general framework for Q-Q plots in the qqmath function, allowing comparison between a sample and any theoretical distribution by specifying the appropriate quantile function (Sarkar, 
2008). qqPlot in the car package also allows for the assessment of non-normal distributions and adds pointwise confidence bands via normal theory or the parametric bootstrap (Fox and Weisberg, 2011). The ggplot2 package provides geom_qq and geom_qq_line, enabling the creation of Q-Q plots with a reference line, much like those created using qqmath (Wickham, 2016). None of these general-use packages allow for easy construction of detrended Q-Q plots.

The qqplotr package extends ggplot2 to provide a complete implementation of Q-Q plots. The package allows for quick construction of all Q-Q plot designs without sacrificing the flexibility of the ggplot2 framework. In the remainder of this paper, we introduce the plotting framework provided by qqplotr and provide multiple examples of how it can be used.

\section{Implementing Q-Q plots in the ggplot2 framework}

qqplotr provides a ggplot2 layering mechanism for $\mathrm{Q}-\mathrm{Q}$ points, reference lines, and confidence bands by implementing separate statistical transformations (stats). In this section, we describe each transformation.

stat_qq_point

This modified version of stat_qq / geom_qq (from ggplot2) plots the sample quantiles against the theoretical quantiles (as in Figure 1). The novelty of this implementation is the ability to create a detrended version of the plotted points. All other transformations in qqplotr also allow for the detrend option. Below, we present a complete call to stat_qq_point and highlight the default values of its parameters:

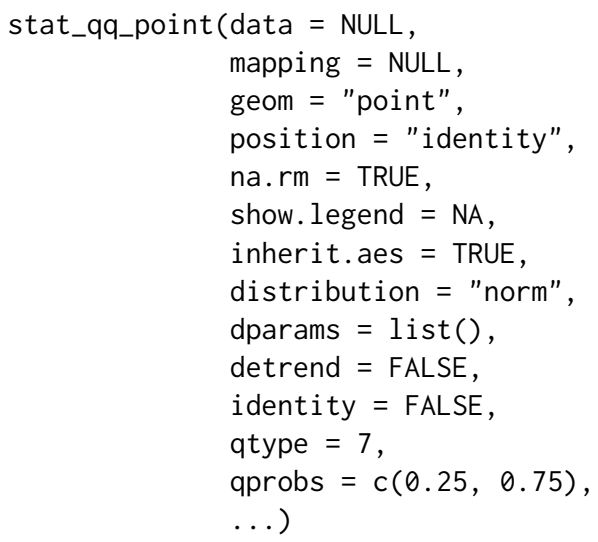

- Parameters such as data, mapping, geom, position, na.rm, show. legend, and inherit. aes are commonly found among several ggplot2 transformations.

- distribution is a character string that sets the theoretical probability distribution. Here, we followed the nomenclature from the stats package, but rather than requiring the full function name for a distribution (e.g., "dnorm"), only the suffix is required (e.g., "norm"). If you wish to provide a custom distribution, then you must first create its density (PDF), distribution (CDF), quantile, and simulation functions, following the nomenclature outlined in stats. For example, to create the "custom" distribution, you must provide the appropriate dcustom, pcustom, qcustom, and rcustom functions. A detailed example is given in the User-provided distributions section.

- dparams is a named list specifying the parameters of the proposed distribution. By default, maximum likelihood etimates (MLEs) are used, so specifying this argument overrides the MLEs. Please note that MLEs are currently only supported for distributions available in stats, so if a custom distribution is provided to distribution, then all of its parameters must be estimated and passed as a named list to dparams.

- detrend is a logical that controls whether the points should be detrended (as in Figure 3), producing ordinary detrended Q-Q plots. For additional details on how to use this parameter and produce the more powerful adjusted detrended Q-Q plots, see the Detrending Q-Q plots section.

- identity is a logical value only used in the case of detrending (i.e., if detrend = TRUE). If identity = FALSE (default), then the points will be detrended according to the traditional Q-Q line that intersects the two data quantiles specified by qprobs (see below). If identity = TRUE, the identity line will be used instead as the reference line when constructing the detrended Q-Q plot. 
- qtype and qprobs are only used when detrend = TRUE and identity = FALSE. These parameters are passed on to the type and probs parameters of the quantile function from stats, both of which are used to specify which quantiles are used to form the Q-Q line.

stat_qq_line

The stat_qq_line statistical transformation draws a reference line in a Q-Q plot.

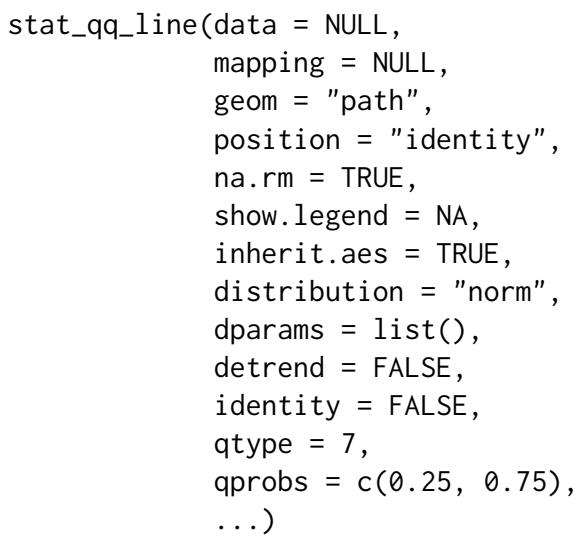

Nearly all of the parameters for stat_qq_line are identical to those for stat_qq_point. Hence, with the exception of identity, all other parameters have the same interpretation. For stat_qq_line, the identity parameter is always used, regardless of the value of detrend. This parameter controls which reference line is drawn:

a) When identity = FALSE (default), the $Q-Q$ line is drawn. By default the Q-Q line is drawn through two points, the .25 and .75 quantiles of the theoretical and empirical distributions. This line provides a robust estimate of the empirical distribution, which is of particular advantage for small samples (Loy et al., 2016).

b) When identity = TRUE, the identity line is drawn. By definition of a Q-Q plot the identity line represents the theoretical distribution.

Both of these reference lines have a special meaning in the context of Q-Q plots. By comparing these two lines we learn about how well the parameters estimated from the sample match the theoretical parameters. For a distributional family that is invariant to linear transformations, the parameters specified in the theoretical distribution only have an effect on the $Q-Q$ line and the $Q-Q$ points. That is, the parameters get shifted and scaled in the plot, but relative relationships do not change aside from a change of scale on the $x$-axis. For other distributions, such as a lognormal distribution, re-specifications of the parameters result in non-linear transformations of the Q-Q line and Q-Q points (see Figure 4 for an example).

stat_qq_band

Confidence bands can be drawn around the reference line using one of four methods: simultaneous Kolmogorov-type bounds, a pointwise normal approximation, the parametric bootstrap (Davison and Hinkley, 1997), or the tail-sensitive procedure (Aldor-Noiman et al., 2013).

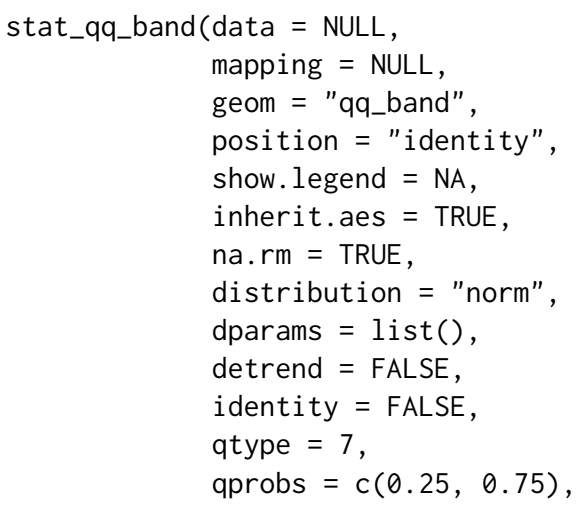



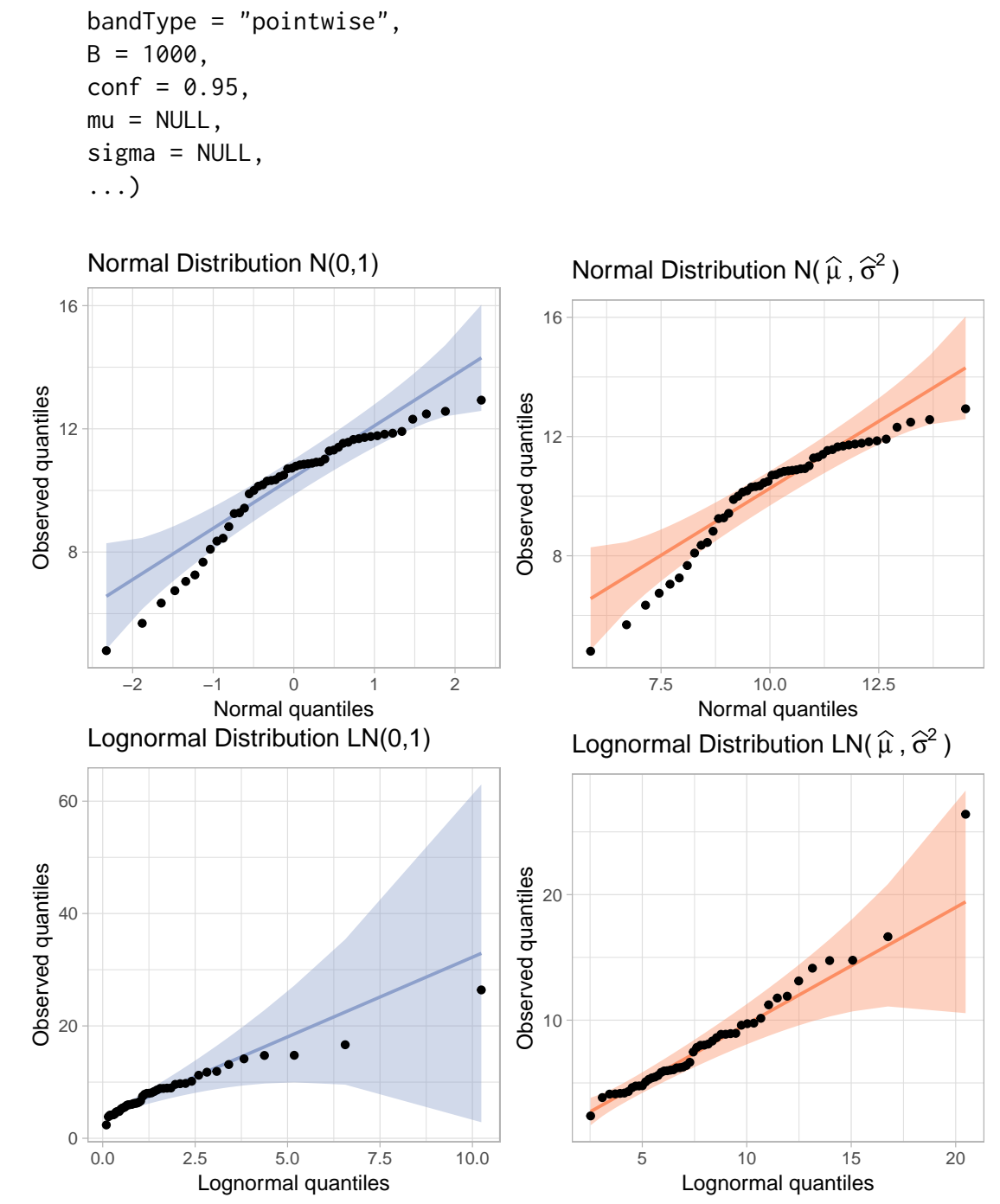

Figure 4: Q-Q plots of a sample of size $n=50$ drawn from a normal distribution setting as theoretical the Standard Normal distribution (top-left) and a normal distribution with ML parameter estimates (top-right). Note how only the scales on the axes change between those plots. The bottom two plots show Q-Q plots of a sample of size $n=50$ drawn from a lognormal distribution. On the left, the mean and variance of the theoretical are 0 and 1, respectively, on the log scale. On the right, ML estimates for mean and variance are used. $95 \%$ pointwise confidence bands are displayed on all Q-Q plots.

- bandType is a character string controlling the method used to construct the confidence bands:

- Simultaneous: Specifying bandType = "ks" constructs simultaneous confidence bands based on an inversion of the Kolmogorov-Smirnov test. For an i.i.d. sample from CDF $F$, the Dvoretzky-Kiefer-Wolfowitz (DKW) inequality (Dvoretzky et al., 1956; Massart et al., 1990) states that $P\left(\sup _{x}|F(x)-\widehat{F}(x)| \geq \varepsilon\right) \leq 2 \exp \left(-2 n \varepsilon^{2}\right)$. Thus, lower and upper $(1-\alpha) 100 \%$ confidence bounds for $\widehat{F}(n)$ are given by $L(x)=\max \{\widehat{F}(n)-\varepsilon, 0\}$ and $U(x)=\min \{\widehat{F}(n)+\varepsilon, 1\}$, respectively. Confidence bounds for the points on a Q-Q plot are then given by $F^{-1}(L(x))$ and $F^{-1}(U(x))$.

- Pointwise: Specifying bandType = "pointwise" constructs pointwise confidence bands based on a normal approximation to the distribution of the order statistics. An approximate $95 \%$ confidence interval for the $i$ th order statistic is $\widehat{X}_{(i)} \pm \Phi^{-1}(.975) \cdot S E\left(X_{(i)}\right)$, where $\widehat{X}_{(i)}$ denotes the value along the fitted reference line, $\Phi^{-1}(\cdot)$ denotes the quantile function for the Standard Normal Distribution, and $S E\left(X_{(i)}\right)$ is the standard error of the $i$ th order statistic.

- Bootstrap: Specifying bandType = "boot" constructs pointwise confidence bands using percentile confidence intervals from the parametric bootstrap. 
- Tail-sensitive: Specifying bandType = "ts" constructs the simulation-based tail-sensitive simultaneous confidence bands proposed by Aldor-Noiman et al. (2013). Currently, tailsensitive bands are only implemented for distribution = "norm".

- $\mathrm{B}$ is a dual-purpose integer parameter. If bandType = "boot", it specifies the number of bootstrap replicates. If bandType $=$ " $t s "$, it specifies the number of simulated samples necessary to construct the tail-sensitive bands.

- conf is a numerical variable bound between 0 and 1 that sets the confidence level of the bands.

- mu and sigma are only used when bandType = "ts". They represent the center and scale parameters, respectively, used to construct the simulated tail-sensitive confidence bands. If either is NULL, then both of the parameters are estimated using robust estimates via the robustbase package (Maechler et al., 2016). Currently, bandType = "ts" is only implemented for distribution $=$ "norm", which is the only distribution discussed by Aldor-Noiman et al. (2013).

\section{Groups in qqplotr}

qqplotr is implemented in accordance with the ggplot2 concept of groups. When the user maps values to aesthetics that explicitly (by using group) or implicitly (such as shape or discrete values of colour, size etc.) introduce groups, the corresponding calculations respect the grouping in the data. All groups are compared to the same distributional family, but the parameters are estimated separately for each of the groups if dparams is not specified (which is the default for all transformations). If the user wants to fit the same distribution (i.e., the same parameter estimates) to each group, then the estimates must be manually calculated and passed to dparams as a named list for each of the desired qqplotr transformations. The use of groups is illustrated in more detail in the BRFSS example section.

\section{Examples}

In this section, we demonstrate the capabilities of qqplotr by providing multiple examples of how the package can be used. We start by loading the package:

library (qqplotr)

\section{Constructing Q-Q plots with qqplotr}

To give a brief introduction on how to use qqplotr and its transformations, consider the urine dataset from the boot package. This small dataset consists of 79 urine specimens that were analyzed to determine if certain physical characteristics of urine (e.g., $\mathrm{pH}$ or urea concentration) might be related to the formation of calcium oxalate crystals. In this example, we focus on the distributional assessment of $\mathrm{pH}$ measurements made on the samples.

We start by creating a normal Q-Q plot of the data. The top-left plot in Figure 5 shows a Q-Q plot comparing the $\mathrm{pH}$ measurements to the normal distribution. The code used to create this plot is shown below. As previously noted, the parameters of the normal distribution are automatically estimated using the MLEs when the parameters are not otherwise specified in dparams. The shaded region represents the area between the normal pointwise confidence bands. As we can see, the distribution of urine $\mathrm{pH}$ measurements is somewhat right-skewed.

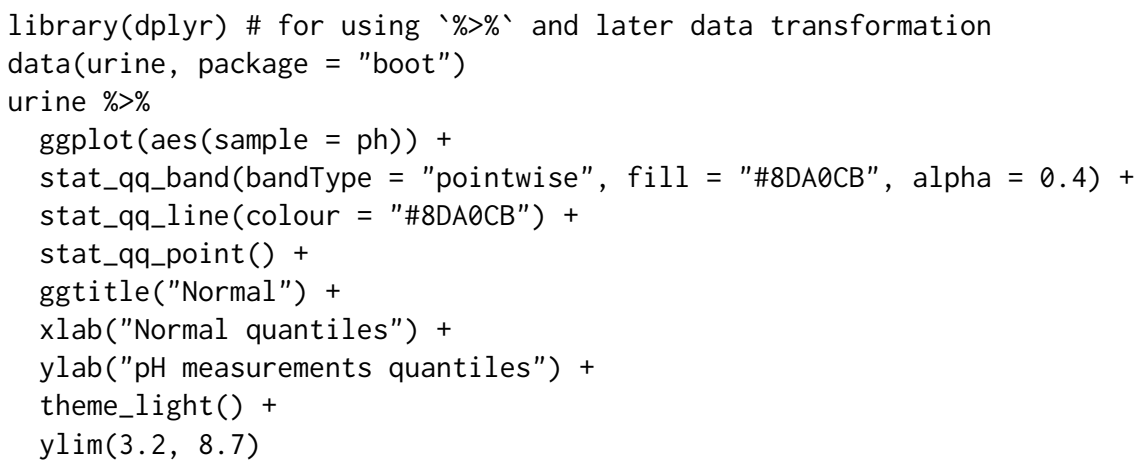

Figure 5 also provides an overview of qqplotr's capabilities:

- The left column displays Q-Q plots with 95\% pointwise confidence bands obtained from a normal approximation. 

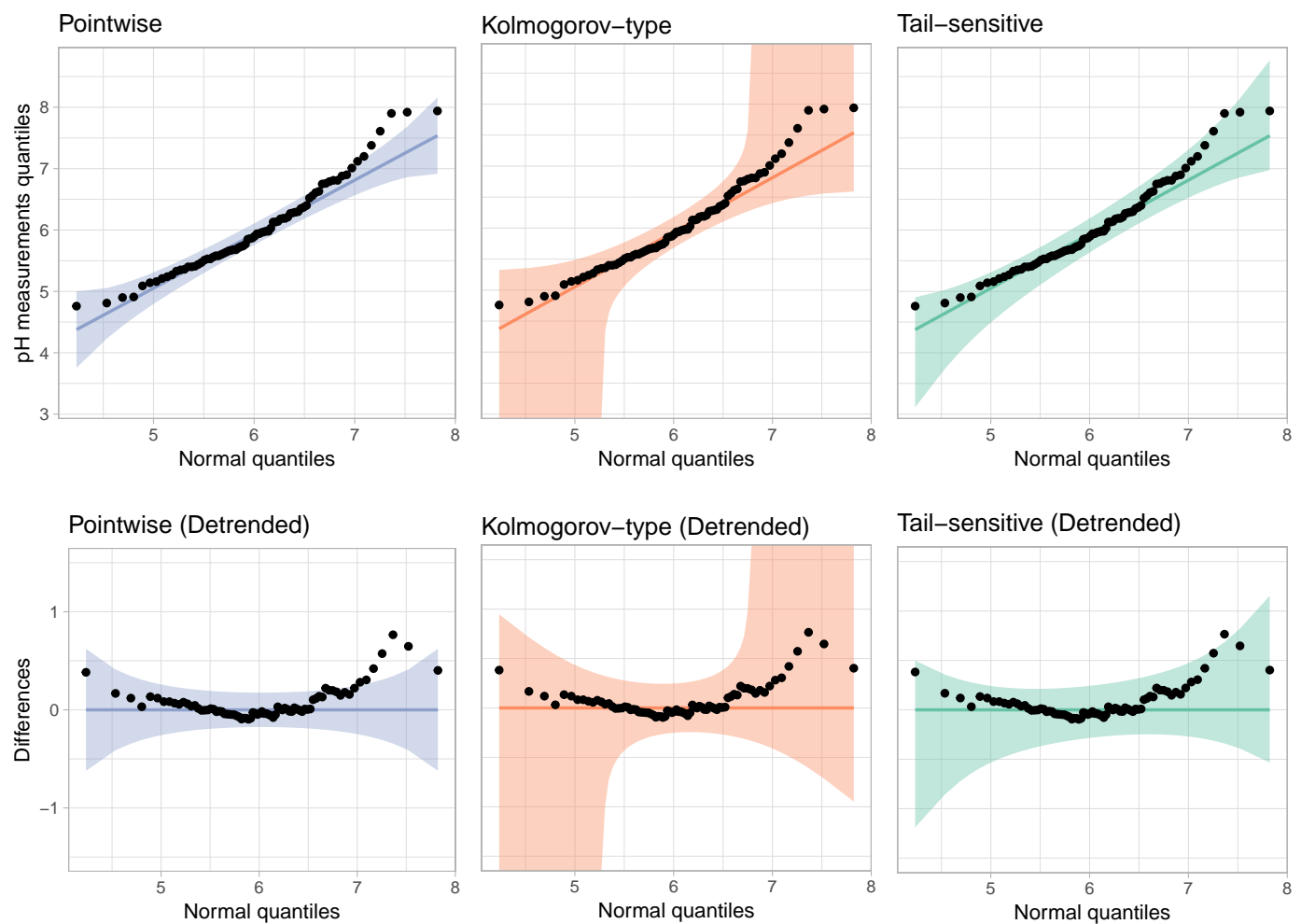

Figure 5: Normal Q-Q plots of $\mathrm{pH}$ measurements from urine samples using different confidence bands. Depending on the type of confidence band used, we come to different conclusions.

- The center column displays Q-Q plots with 95\% Kolmogorov-type simultaneous confidence bands.

- The right column displays Q-Q plots with 95\% tail-sensitive simultaneous confidence bands. Notice that these are substantially narrower in the tails than the Kolmogorov-type bands.

- The bottom row shows the detrended versions of the Q-Q plots in the top row.

\section{User-provided distributions}

Using the capabilities of qqplotr with the distributions implemented in stats is relatively straightfoward, since the implementation allows you to specify the suffix (i.e., distribution or abbreviation) via the distribution argument and the parameter estimates via the dparams argument. However, there are times when the distributions in stats are not sufficient for the demands of the analysis. For example, there is no left-skewed distribution listed aside from the beta distribution, which has a restrictive support. User-coded distributions, or distributions from other packages, can be used with qqplotr as long as the distributions are defined following the conventions laid out in stats. Specfically, for some distribution there must be density/mass ( $d$ prefix), CDF ( $p$ prefix), quantile ( $q$ prefix), and simulation ( $r$ prefix) functions. In this section, we illustrate the use of the smallest extreme value distribution (SEV).

To qualify for the 2012 Olympics in the men's long jump, athletes had to meet/exceed the 8.1 meter standard or place in the top twelve. During the qualification events, each athlete was able to jump up to three times, using their best (i.e., longest) jump as the result. Figure 6 shows a density plot of the results, which is clearly left skewed.

We start by loading the longjump dataset included in qqplotr and removing any NAs:

data ("longjump", package = "qqplotr")

longjump <- na.omit (longjump)

Next, we define the suite of distributional functions necessary to utilize the SEV distribution.

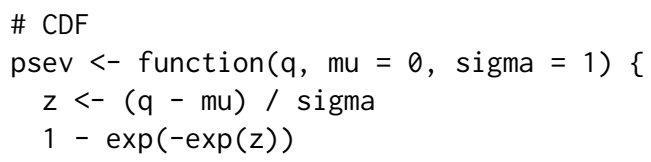




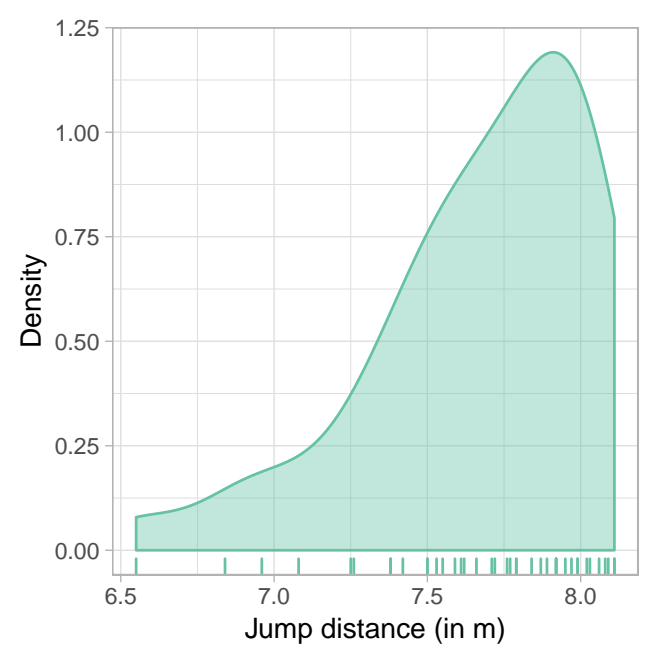

Figure 6: Density and rug plot of the 2012 men's long jump qualifying round. The distances are clearly left skewed.

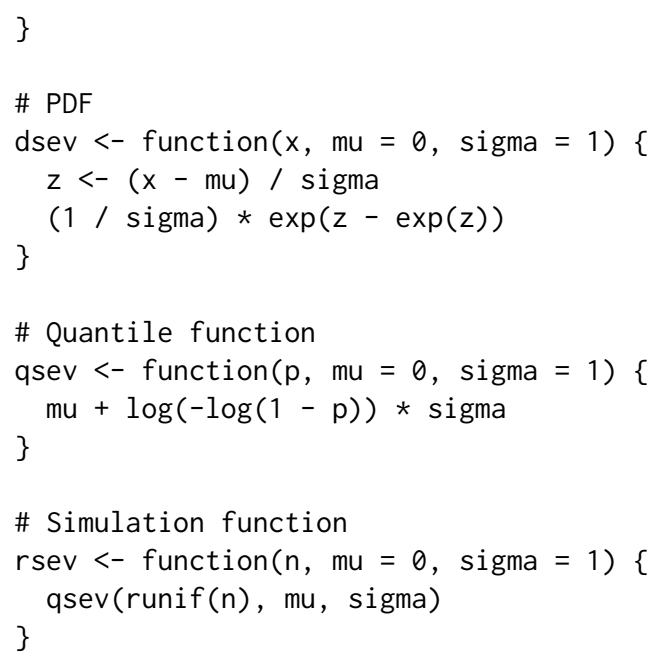

With the *sev distribution functions in hand, we can create a Q-Q plot to assess the appropriateness of the SEV model (Figure 7). The Q-Q plot shows that the distances do not substantially deviate from the SEV model, so we have found an adequate representation of the distances. The code used to create Figure 7 is given below:

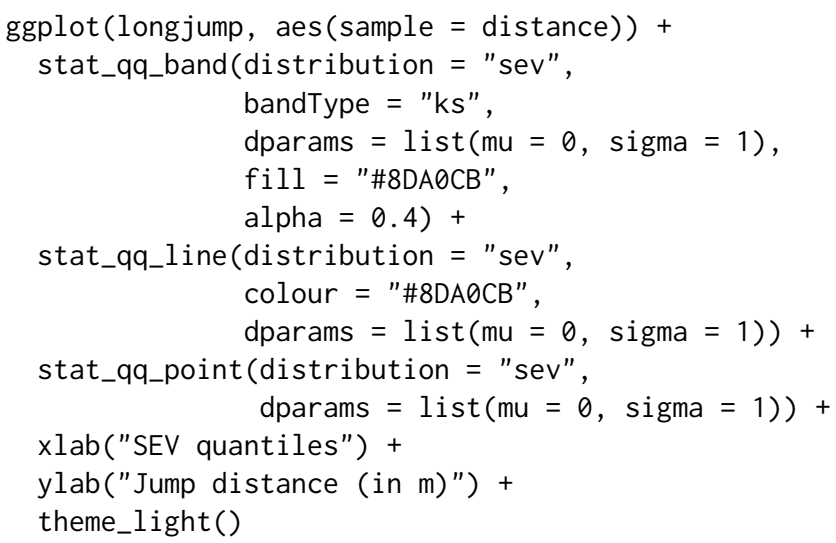

\section{Detrending Q-Q plots}

To illustrate how to construct an adjusted detrended Q-Q plot using qqplotr, consider detrending Figure 7. This is done by adding the argument detrend = TRUE to stat_qq_point, stat_qq_line, and stat_qq_band. To adjust the aspect ratio to ensure that vertical and horizontal distances are on the 


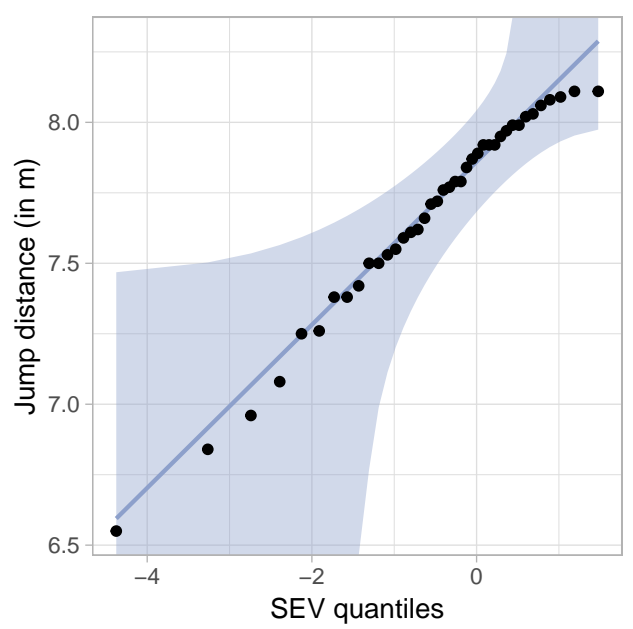

Figure 7: Q-Q plot comparing the long jump distances to the standard SEV distribution with 95\% simultaneous confidence bands. The SEV distribution appears to adequately model the distances.

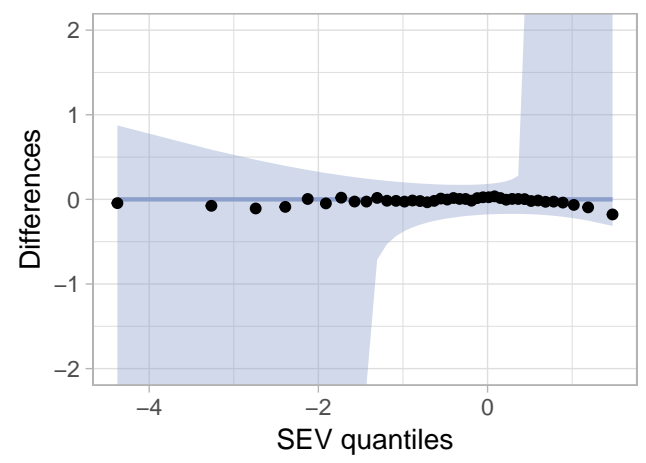

Figure 8: An adjusted detrended Q-Q plot assessing the appropriateness of the SEV distribution for the long jump data.

same scale we further add coord_fixed $($ ratio $=1)$. We leave it to the user to adjust the $y$-axis limits on a case-by-case basis. The full command to construct Figure 8 is given below:

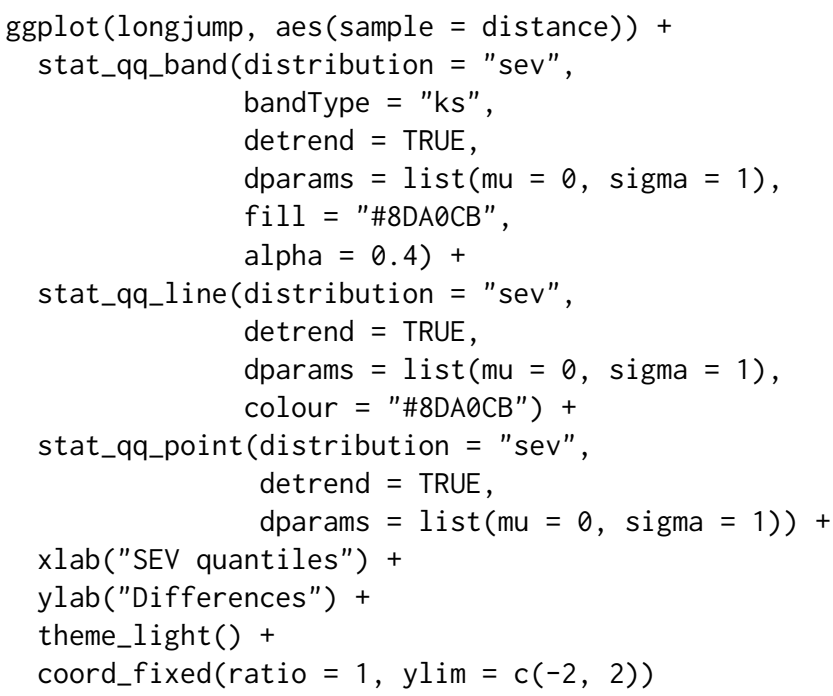

\section{BRFSS example}

The Center for Disease Control and Prevention runs an annual telephone survey, the Behavioral Risk Factor Surveillance System (BRFSS), to track "health-related risk behaviors, chronic health conditions, and use of preventive services" (Centers for Disease Control and Prevention, 2014). Close to half a 
million interviews are conducted each year. In this example, we focus on the responses for Iowa in 2012. The data set consists of 7166 responses across 359 questions and derived variables. To further illustrate the functionality of qqplotr, we focus on assessing the distributions of Iowan's heights and weights.

Figure 9 shows two Q-Q plots constructed from a sample of 200 men and 200 women drawn from the overall number of responses. On the left-hand side, individuals' heights are displayed in a Q-Q plot comparing raw heights to a normal distribution. We see that the distributions for both men and women show horizontal steps: this indicates that the distributional assessement is heavily dominated by the discreteness in the data, as most respondents provided their height to the nearest inch. On the right-hand side of Figure 9, we use jittering to remedy this situation. That is, we add a random number generated from a random uniform distribution on \pm 0.5 inch to the reported height, as shown in the code below:

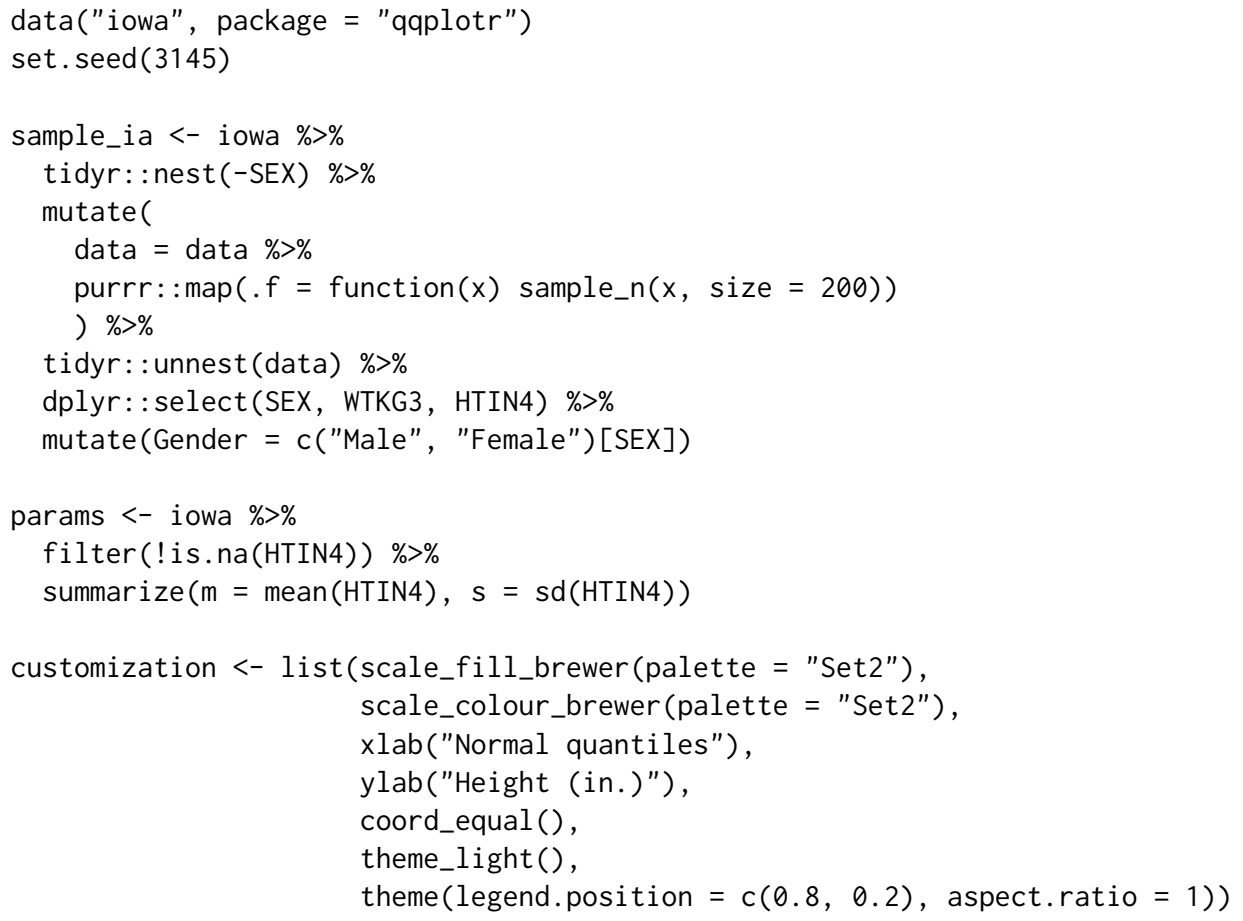

Notice that the same theoretical normal distribution was fit to both genders as specified in dparams. If we had used the default, then the MLEs for each gender would be used. As a result, we would be comparing the two genders over a different range of theoretical quantiles. By explicity providing parameter estimates for the mean and standard deviation via dparams, we force the Q-Q plots to use the same $x$-coordinates (theoretical quantiles), which is more useful when comparing the distribution of these groups.

As seen in Figure 9, by using jittering we diminish the effect that discreteness has on the distribution 

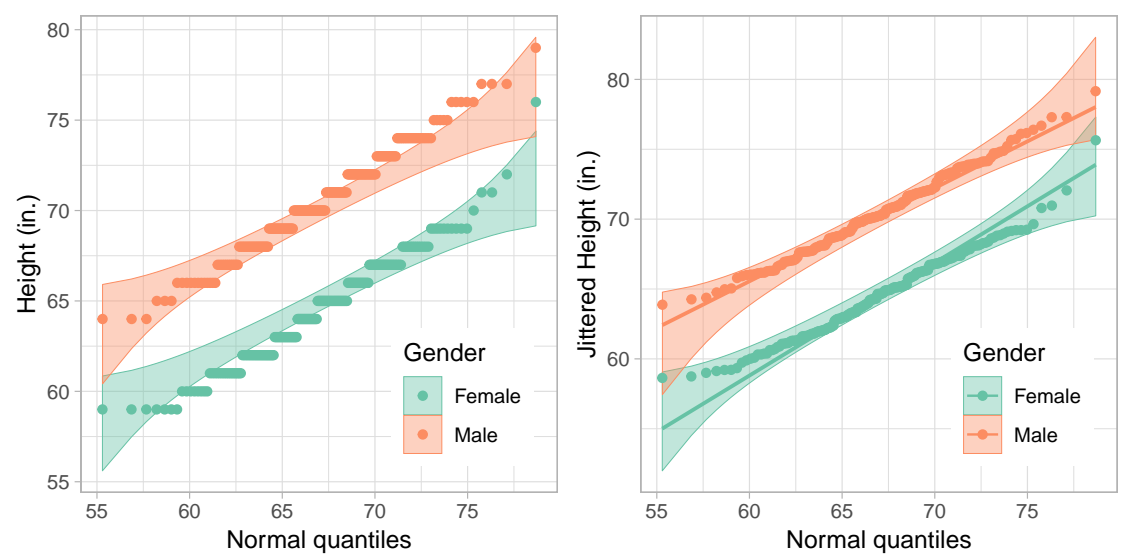

Figure 9: $Q-Q$ plots comparing the raw (left) and jittered (right) heights to a normal distribution for a sample of 200 men and 200 women. The distribution on the left is dominated by the discreteness of the data. On the right, using a normal distribution to model people's height is not completely absurd, except for a few extreme outliers.

Table 1: Summary of Iowa residents' heights and weights with corresponding standard deviations by gender and for the total population.

\begin{tabular}{lrrrr}
\hline SEX & mean height (in) & sd (in) & mean log weight $(\mathrm{kg})$ & sd $(\mathrm{kg})$ \\
\hline Male & 70.55 & 2.97 & 9.10 & 0.20 \\
Female & 64.51 & 2.91 & 8.89 & 0.23 \\
Total & 66.99 & 4.18 & 8.98 & 0.24 \\
\hline
\end{tabular}

and brings the observed distribution much closer to a normal distribution. Unsurprisingly, the resulting distributions have different means (women are, on average, 6 inches shorter than men in this data set). Interestingly, the slope of the two genders is similar, indicating that the same scale parameter fits both genders' distributions (the standard deviation of height in the data set is 2.97 inch for men and 2.91 inch for women, see Table 1).

Unlike respondents' heights, their weights do not seem to be normally distributed. Figure 10 shows two Q-Q plots of these data. For both, distributional parameters are estimated separately for each group. This means that for each group we compare against its theoretical distribution shown as the identity line. The Q-Q plot on the left compares raw weights to a normal distribution. We see that tails of the observed distribution are heavier than expected under a normal distribution. On the right, weights are log-transformed. We see that using a normal distribution for each gender appears to be reasonable, with the exception of a few extreme outliers. The code used to create Figure 10 is shown below:

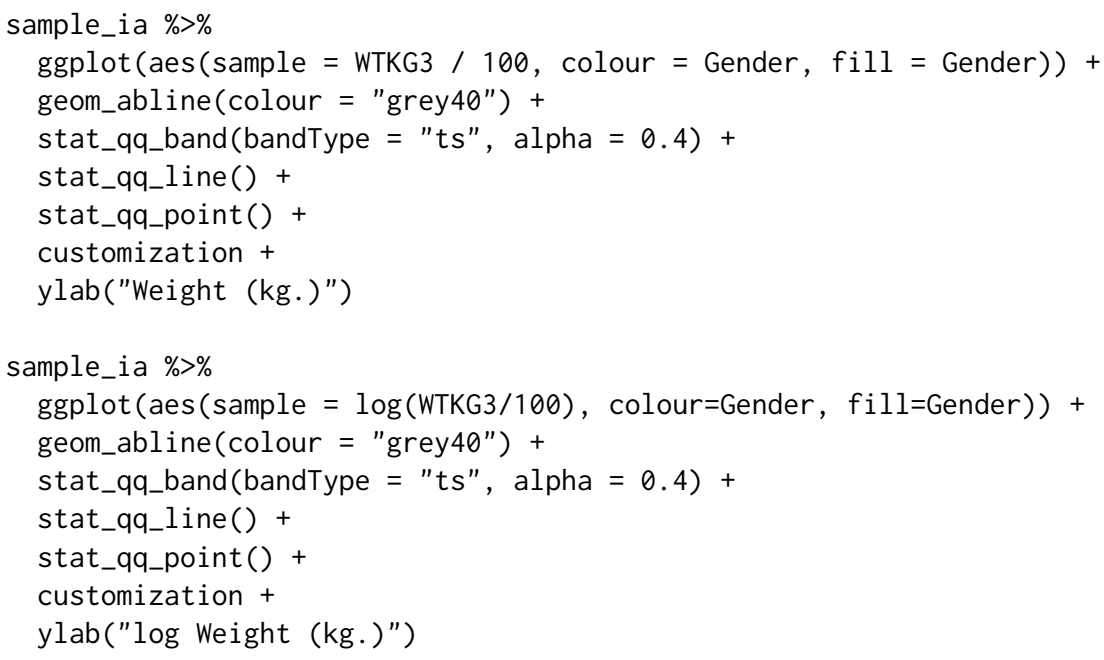

Instead of log-transforming the observed weights, we can change the theoretical distribution to a lognormal. Figure 11 shows two lognormal Q-Q plots, one for each gender. Note the MLEs are used to 

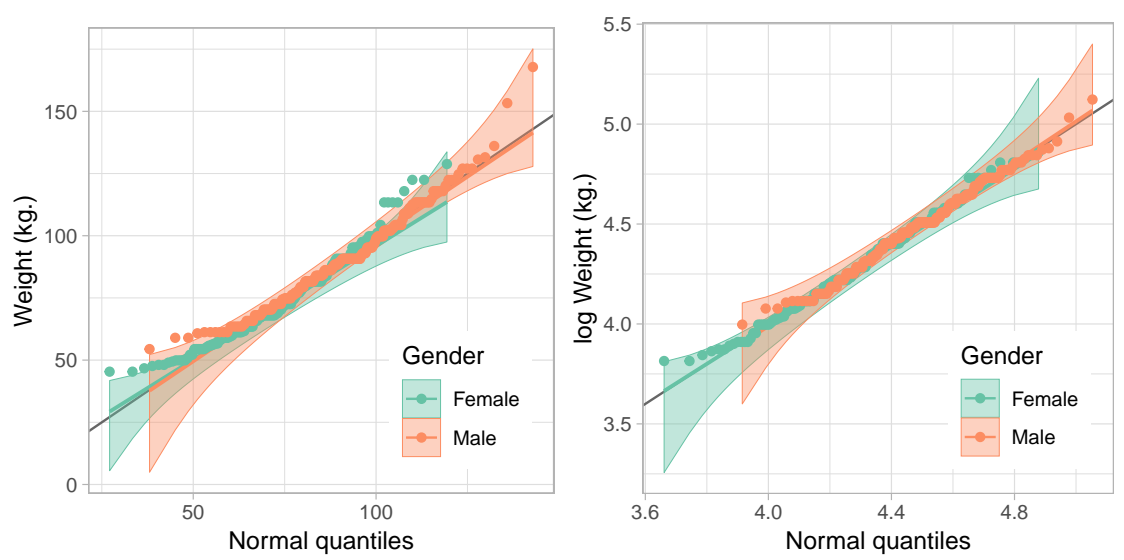

Figure 10: Q-Q plots comparing weights to a normal distribution for a sample of 200 men and 200 women. Unlike people's height, weight seems to be right skewed with some additional outliers in the left tail (left plot). On the right, weight was log-transfomed before its distribution is compared to a theoretical normal.

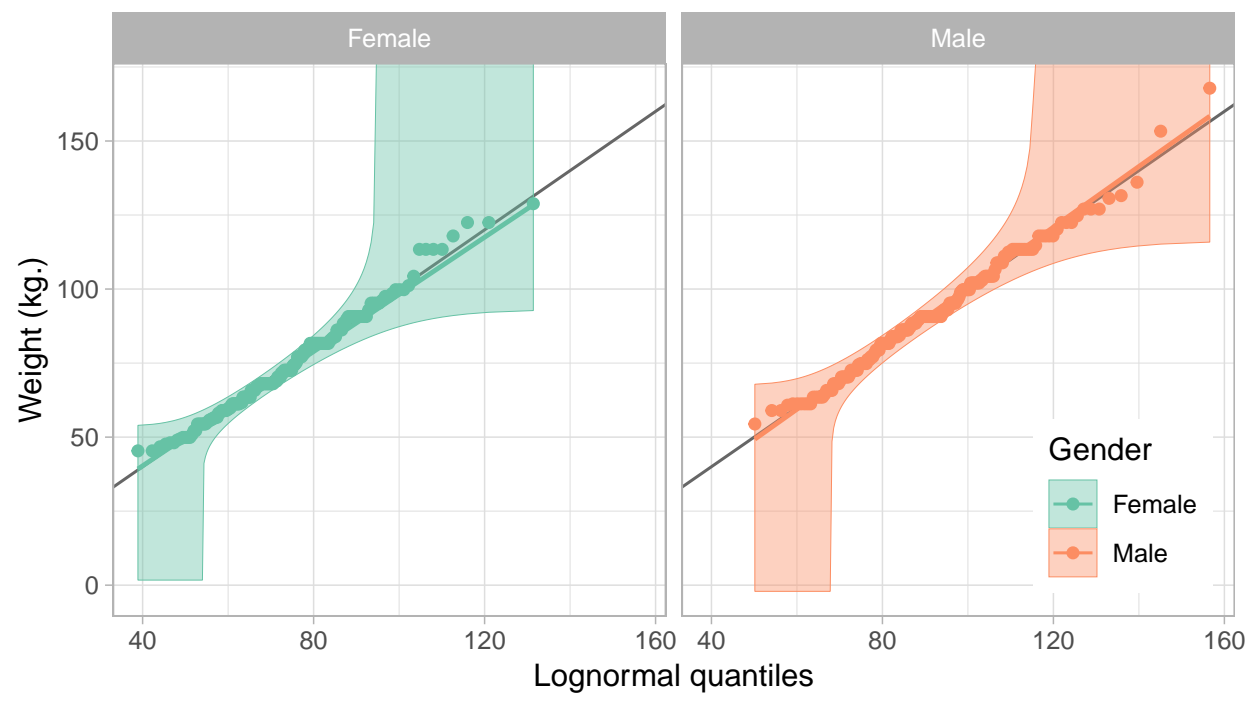

Figure 11: Q-Q plots comparing weights to a lognormal distribution for a sample of 200 men and 200 women. The parameters are estimated separately for each gender to specify the lognormal reference distribution for each gender.

parameterize the lognormal distribution for each group since dparams is not specified:

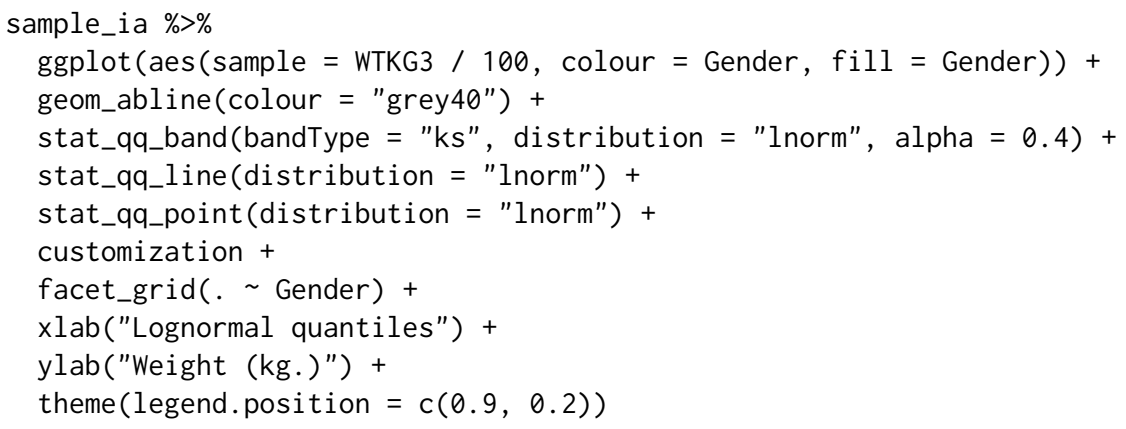

\section{Discussion}

This paper presented the qqplotr package, an extension of ggplot2 that implements Q-Q plots in both the standard and detrended orientations, along with reference lines and confidence bands. The examples illustrated how to create Q-Q plots for non-standard distributions found outside of the stats 
package, how to create detrended Q-Q plots, and how to create Q-Q plots when data are grouped. Further, in the BRFSS example, we illustrated how jittering can be used in Q-Q plots to better compare discretized data to a continuous distribution.

While qqplotr provides a complete implementation of the Q-Q plot, there is room for development in future versions. For example, Q-Q plots are members of the larger probability plotting family, so future versions of qqplotr will likely include additional members of that family.

Finally, we have made design choices in qqplotr that we believe are in line with best practices for distributional assessment, but the implementation is flexible enough to allow for easy customization. For example, maximum likelihood is used to estimate the parameters of the proposed model, but if outliers are present robust estimators may be desirable, such as when comparing the empirical distribution to a normal distribution. In this scenario, robust estimates of the location and scale can be obtained using the robustbase package (Maechler et al., 2016), and specified using the dparams parameter directly. This is especially useful if you wish to use the parametric bootstrap to build confidence bands. Similarly, stat_qq_line implements two types of reference lines: the identity line, and the traditional Q-Q line that passes through two quantiles of the distributions, such as the first and third quartiles. While those are the most conventionally used reference lines, alternative ones can be quickly implemented using ggplot2: :geom_abline by specifying the slope and intercept. By default, the Q-Q line is used; however, this is not always the most appropriate choice. In order to test whether the data follow a specific distribution, the reference line should be used rather than using the data twice: once to estimate the parameters, and once for comparison.

\section{Bibliography}

S. Aldor-Noiman, L. D. Brown, A. Buja, W. Rolke, and R. A. Stine. The power to see: A new graphical test of normality. The American Statistician, 67(4):249-260, 2013. URL https://doi .org/10.1080/ 00031305.2013 .847865$. [p251, 253]

Centers for Disease Control and Prevention. Behavioral risk factor surveillance system. https: //www.cdc.gov/brfss/about/index.htm, 2014. Accessed: 2017-11-1. [p256]

W. S. Cleveland and R. McGill. Graphical perception: Theory, experimentation, and application to the development of graphical methods. Journal of the American Statistical Association, 79(387):531-554, 1984. ISSN 01621459. URL https://doi.org/10.2307/2288400. [p249]

A. C. Davison and D. V. Hinkley. Bootstrap Methods and their Application. Cambridge University Press, Cambridge, 1997. ISBN 9780521574716. [p251]

A. Dvoretzky, J. Kiefer, and J. Wolfowitz. Asymptotic minimax character of the sample distribution function and of the classical multinomial estimator. Annals of Mathematical Statistics, 27(3):642-669, 9 1956. URL https://doi .org/10.1214/aoms/1177728174. [p252]

J. Fox and S. Weisberg. An R Companion to Applied Regression. Sage, Thousand Oaks CA, second edition, 2011. ISBN 9781412975148. [p250]

A. Loy, L. Follett, and H. Hofmann. Variations of Q-Q plots: The power of our eyes! The American Statistician, 70(2):202-214, 2016. URL https://doi.org/10.1080/00031305.2015.1077728. [p249, 251]

M. Maechler, P. Rousseeuw, C. Croux, V. Todorov, A. Ruckstuhl, M. Salibian-Barrera, T. Verbeke, M. Koller, E. L. T. Conceicao, and M. Anna di Palma. robustbase: Basic Robust Statistics, 2016. URL http://robustbase.r-forge.r-project.org/. R package version 0.92-7. [p253, 260]

P. Massart et al. The tight constant in the Dvoretzky-Kiefer-Wolfowitz inequality. The Annals of Probability, 18(3):1269-1283, 1990. URL https://doi .org/10.1214/aop/1176990746. [p252]

N. Robbins. Creating More Effective Graphs. Wiley, Hoboken, 2005. ISBN 0-471-27402-x. [p249]

D. Sarkar. lattice: Multivariate Data Visualization with R. Springer, New York, 2008. ISBN 978-0-38775968-5. [p249]

S. Vander Plas and H. Hofmann. Signs of the sine illusion - and why we need to care. Journal of Computational and Graphical Statistics, 25:1170-1190, 2015. URL https://doi .org/10.1080/10618600. 2014.951547. [p249]

H. Wickham. ggplot2: Elegant Graphics for Data Analysis. Springer-Verlag New York, 2016. ISBN 978-3-319-24277-4. [p250] 
M. B. Wilk and R. Gnanadesikan. Probability plotting methods for the analysis of data. Biometrika, 55 (1):1-17, 1968. URL https://doi .org/10.2307/2334448. [p248]

\section{Acknowledgements}

This work was partially funded by Google Summer of Code 2017. We thank the reviewers for their helpful comments and suggestions.

Alexandre Almeida

University of Campinas

Institute of Computing

Campinas, Brazil 13083-852

almeida.xan@gmail.com

Adam Loy

Carleton College

Department of Mathematics and Statistics

Northfield, MN 55057

ORCiD: 0000-0002-5780-4611

aloy@carleton.edu

Heike Hofmann

Iowa State University

Department of Statistics

Ames, IA 50011-1210

ORCiD: 0000-0001-6216-5183

hofmann@iastate.edu 